
de management

\title{
L'« usager » télé-visé : TGV bloqués, caténaires gelées et médiatisation de l'information en situation perturbée
}

The Television "User:" Train Disruptions, Frozen Electrical Lines, and the Media Treatment of Information in a Crisis Situation

Jacques Noyer

\section{OpenEdition}

Journals

Édition électronique

URL : http://journals.openedition.org/edc/1141

DOI : 10.4000/edc. 1141

ISSN : 2101-0366

Éditeur

Université Lille-3

\section{Édition imprimée}

Date de publication : 1 mars 2001

Pagination : 105-128

ISBN : 2-9514961-1-7

ISSN : $1270-6841$

\section{Référence électronique}

Jacques Noyer, «L'« usager » télé-visé : TGV bloqués, caténaires gelées et médiatisation de l'information en situation perturbée », Études de communication [En ligne], 23 | 2001, mis en ligne le 10 janvier 2010, consulté le 03 mai 2019. URL : http://journals.openedition.org/edc/1141 ; DOI : 10.4000/ edc. 1141

Ce document a été généré automatiquement le 3 mai 2019.

(c) Tous droits réservés 


\title{
L'« usager » télé-visé : TGV bloqués, caténaires gelées et médiatisation de l'information en situation perturbée
}

\author{
The Television "User:" Train Disruptions, Frozen Electrical Lines, and the Media \\ Treatment of Information in a Crisis Situation
}

Jacques Noyer

1 La question de la place et du rôle de l'« usager $»^{1}$ dans les services publics ${ }^{2}$ a généré depuis le début du siècle (Daviet, 1996, Horellou, 1996), et en particulier depuis la fin de la seconde guerre mondiale (Warin, 1992, Chauvière-Godbout, 1992), de nombreux travaux de recherche qui, issus de champs disciplinaires multiples, offrent un panorama large des modes d'approche possibles de cette figure.

2 Sous un angle plus particulièrement communicationnel, cette question est fréquemment abordée au travers de démarches questionnant les modes de réalisation du service : ce qui se joue dans la relation de demande et de prestation constitue le cœur de l'analyse. Elle n'est que très rarement traitée sous l'angle, plus indirect, des médiations ${ }^{3}$ sociales qui, parallèlement aux pratiques d'accomplissement du service, contribuent à forger les représentations que nous avons des services publics et de leurs qualités ou défauts, de l'« usager » et de ses modes de traitement dans le cours des différents types de service.

3 Le mode d'approche que nous envisageons ici consistera non pas à focaliser sur le face à face « usager "-agent mais à tenter de comprendre ce qui, socialement, en transparait audelà de la relation de personne à personne. Ce que les discours publics ultérieurs à une relation de service, et s'attachant à l'analyser, dégagent comme caractéristiques centrales de cette relation inter-individuelle et, à travers elle, de l'«usager " à l'institution prestataire constituera l'objet central de notre questionnement. 
C'est dire que l'on ne saurait ignorer ce que la micro-sociologie et les analyses interactionnistes ont pu, à ce propos, permettre d'utilement cadrer pour comprendre ce qui se joue à de multiples niveaux dans ce type d'« échange». On se souviendra, en particulier, de l'importance de certaines de ces recherches portant sur des lieux spécifiques (Grosjean, 1993, Lacoste, 1995), sur certaines opérations (Cosnier-Picard, 1992, Heath, 1993, Borzeix, 1995-a, Borzeix, Fisher, De Fornel, Lacoste, 1995). Les apports déterminants de ces travaux constituent, d'ailleurs, sur de nombreux aspects du processus de communication des éléments de référence indispensables à l'élaboration de façons complémentaires d'appréhender la relation de service et ce qui la rend possible :

- notamment, tout ce qui, en termes d'organisation, fonctionne comme l'amont de la relation et en permet la réalisation;

- mais aussi ce qui, en aval, apparaît comme discours, plus ou moins socialisés, sur les pratiques de tel ou tel lieu de service au public et dont la diffusion publique n'est pas sans avoir des incidences sur la perception générale que l'on a de l'institution. Certaines des stratégies de ces entreprises publiques et certaines des évolutions de service qu'elles mettent en place peuvent, d'ailleurs, apparaître comme des conséquences de ce que le discours évaluatif de l'« usager", sous ses diverses formes et selon ses divers modes de collecte, a permis de faire affleurer ${ }^{4}$. Modernisation des services publics et accentuation de la prise en compte de l'«usager» et de ses discours ont partie liée et déterminent le « pilotage par l'aval » dont parle Jeannot (1998). Il y a donc, selon nous, un intérêt à observer attentivement les espaces d'expression de l'«usager» et, notamment, ceux qui, par la publicisation qu'ils autorisent, en renforcent sans doute l'audibilité et la prise en compte institutionnelle.

\section{Relation de service - relation de service médiatisée}

5 Si l'expérience directe de la relation de service que nous avons dans le quotidien avec les agents de la SNCF, des CAF, de la Poste... est susceptible de gouverner la représentation que nous avons du service - et de sa qualité - dans ces différentes institutions, nous pouvons raisonnablement faire l'hypothèse qu'au-delà de la relation de service ellemême, les différentes " arènes publiques " (Quéré, 1995) où ces questions sont débattues et publicisées ne sont pas sans jouer un rôle sur les modes de perception que nous avons de ces institutions et des services qu'elles proposent. La question est, certes, ici celle de la possibilité d'accès de l'« usager » à ces lieux où sont mis en débat public les questions qui le concernent. En dehors des lieux institutionnels où il peut, par le biais d'associations ou de comités, avoir un accès - toujours très limité et dont la représentativité est toujours fortement questionnée - l'« usager » reste, malgré la volonté croissante d'en faire un coproducteur, davantage agi qu'acteur, objet d'une visée plutôt que véritable partie prenante d'un processus.

Il nous semble pourtant qu'il y a, dans la société, un espace où la question de l'« usager » si ce n'est sa parole - a une place importante quoique fréquemment inaperçue parce que disséminée sur une quantité de terrains qui, pouvant aller des télécommunications aux transports, de l'éducation aux questions de sécurité publique ..., ne laisse pas transparaître a priori les constantes de cette figure de l'« usager » : nous voulons parler des médias, auxquels tout "usager»/client de n'importe quel service public a, par définition, potentiellement accès et qui s'attachent à donner à l'« usager " un espace d'expression « face à » certains (dys)fonctionnements du service, certaines évolutions de 
celui-ci et aux questions que cela soulève. L'« usager » est alors dé-placé du strict rapport d'usage d'un service à un rapport d'observation-analyse de ce qui perturbe ou questionne cet usage. C'est ce dé-placement et les discours qui l'accompagnent que nous souhaiterions prendre en compte ici :

- A quelles conditions y a-t-il médiatisation de ces questions?

- A quelles paroles les médias donnent-ils accès lorsqu'il s'agit de médiatiser les questions relatives aux services publics?

- A quelles figures de l'« usager » accorde-t-on un espace d'expression privilégié ?

- Quelle relation entre l'institution et ceux qui font usage des services qu'elle propose (ré)élabore-t-on?

7 On peut faire l'hypothèse que l'image que l'« usager » se fait du service dans tel ou tel service public n'émane pas seulement de son expérience directe de face à face avec l'institution mais des conjonctions de discours, à teneurs évaluatives diverses, auxquels les médias donnent une visibilité particulière. Le matériau discursif complexe élaboré par le travail médiatique aboutit à construire, à côté de la relation de service, une « relation de service médiatisée » qui, selon nous, participe de l'image que se fait l'« usager » des services publics et qui pourra, dans certains cas, guider pour partie la relation qu'il entretiendra ultérieurement avec telle ou telle institution. Il importe, par conséquent, de prendre en compte, entre autres dimensions, les rapports qu'entretiennent les services publics avec cet élément essentiel de l'espace public que constituent les médias.

\section{Quelles logiques de médiatisation?}

Si l'on considère que tout « usager » des services publics est, peu ou prou, téléspectateur, auditeur, lecteur de journaux ou de magazines et, parfois, l'un et l'autre à la fois, sans doute l'attention à l'égard de l'« usager ", de ses préoccupations et de ses intérêts, est-elle un moyen de traduire concrètement le souci, fréquemment affirmé par les médias quels qu'ils soient, de plus grande proximité avec leurs publics. N'affirment-ils pas ainsi leur volonté de donner la parole à ceux qui ne l'ont guère et en qui chacun pourra se reconnaître ? En ce sens, l'« usager » (des services publics) médiatisé et l'« usager » des médias se rejoignent : la parole ordinaire - journalistiquement perçue comme légitime puisque témoignant d'un vécu - est haussée en parole représentative, transposable à quelques millions d'exemplaires et, à tout le moins, reconnaissable comme une expérience déjà vécue à un moment ou à un autre, ou pouvant potentiellement l'être. $\mathrm{Ce}$ que l'écran ou la page de journal me renvoie, ainsi, c'est une part de ma face sociale, déployée en variations multiples (protestataire, propositionnelle, célébratrice...) plus ou moins investissables et que le média mobilise comme outil de son propre lien au public. L'acte de médiatiser de telles questions et d'en manifester le caractère partageable par tous coïncide, par ce biais, avec celui de fédérer du public, opération dont on admettra qu'elle n'est pas complètement secondaire pour un média...

Le problème du lien au public s'accompagne sans doute d'un niveau complémentaire de questionnement : à quelles occasions les médias - et notamment la télévision - abordentils les questions liées aux services publics? Quels sont les éléments déclencheurs de médiatisation de ces questions? Poser ces questions par rapport au terrain spécifique des services publics amène inévitablement à évoquer les pratiques professionnelles usuelles des journalistes, dont la médiatisation des questions touchant aux services publics ne constitue qu'un point d'application particulier. On peut considérer, à cet égard, que les 
pratiques routinisées de sélection en vigueur chez les journalistes sur de nombreux thèmes sociaux fonctionnent également quand il s'agit de traiter de questions relatives aux services publics ${ }^{5}$.

- De ce point de vue, toute évolution par rapport à un état antérieur, notamment lorsque cette évolution est programmée et inscrite sur l'agenda des politiques publiques, apparaît comme un critère généralement suffisant pour mobiliser, du moins ponctuellement, les médias sur cette évolution. La réforme des 35 heures et les négociations qu'elle a entraînées dans la fonction publique ont été, récemment, un élément de stimulation du traitement médiatique de questions relevant, pour partie, de la nature, des conditions et de la qualité du service proposé au public (programmation).

- Lorsque de telles évolutions du cadre d'exercice de la fonction publique s'accompagnent de conflits, comme on a pu le voir récemment à la Poste ou au Ministère de l'Économie et des Finances, le double caractère d'évolution d'un état et d'écart par rapport à une norme (ici, la négociation) se traduisant par un conflit social, est générateur de séquences plus ou moins longues, plus ou moins fréquentes de médiatisation où il est rare que ne soit pas évoqué l'« usager », notamment sur le mode de la thématique récurrente de la "prise en otage » (programmation + non-programmation).

- On peut enfin considérer que toute situation d'écart par rapport à une norme de fonctionnement sera, à elle seule, potentiellement génératrice de médiatisation: toute rupture de service est susceptible d'apparaitre, aux yeux des journalistes, comme un décalage suffisant par rapport à une norme pour agir comme déclencheur de médiatisation. Par-delà le traitement de l'urgence, de la «crise » et de sa gestion en tant qu'occurrence ponctuelle, c'est toute la question de l'organisation et de ses performances qui se trouvera questionnée au travers d'un certain nombre d'angles de prise - généralement récurrents d'une crise à une autre. Les tempêtes de fin 1999 ont été l'occasion, par exemple, de faire état non seulement des dégâts occasionnés par les vents exceptionnels mais aussi de rendre compte des niveaux de performance des différents services publics, reliant ceux-ci aux compétences organisationnelles des institutions à mobiliser des équipes, des moyens... La comparaison des degrés d'investissement et des capacités de mobilisation est, dans ce cas de figure, une dimension implicite régulière où le service à l'« usager » (et les espaces de parole qu'on lui accorde) sert fréquemment d'élément de référence central (non-programmation) ${ }^{6}$.

\section{Janvier 1997 : une rupture de service médiatisée}

On a coutume de dire, dans les salles de rédaction des médias et dans les lieux de formation au journalisme, que l'information, ce sont « les trains qui arrivent en retard». Façon de désigner l'écart, l'anomalie, la dérogation comme principes d'orientation de l'attention médiatique et la perturbation d'un système, d'un lieu, d'un fonctionnement comme moments particulièrement propices à un travail d'information. La métaphore ferroviaire est comme l'image communément acceptée d'un principe qui guide les attitudes et conceptions des journalistes; au risque, d'ailleurs, de ne plus laisser apparaitre une occurrence digne d'attention médiatique comme une anomalie mais comme quelque chose qui, du fait même d'être publiquement pointé, est perçu comme une nouvelle "norme». Mais lorsque les trains non-métaphoriques se mettent à véritablement arriver en retard, c'est-à-dire, pour une partie d'entre eux dans le cas qui nous préoccupe, à ne plus circuler du tout, qu'en est-il de l'information proposée, des discours mobilisés pour l'élaborer, des cadres d'analyse construits pour en faire état? 
11 Dans la journée du 2 janvier 1997, à la veille d'un week-end de retour de vacances scolaires, une vague de froid s'abat sur toute l'Europe, paralysant une partie importante du trafic routier et aérien. Dans la vallée du Rhône, c'est le givre déposé sur les caténaires des voies ferrées qui bloque le trafic du TGV sud-est. Plusieurs milliers de voyageurs passent la nuit du 2 au 3 dans des TGV immobilisés entre Lyon et Marseille ou dans des centres d'hébergement de fortune.

12 Cette situation et les conséquences qu'elle entraîne présentent, par rapport au type d'axe d'étude que nous nous fixons, plusieurs intérêts :

- la situation de perturbation est suffisamment grave (en extension géographique et en quantité de personnes touchées) pour qu'elle génère une "couverture " médiatique développée sur plusieurs journées, aussi bien à la télévision que dans la presse. Elle rend possible, par conséquent, un travail à propos des discours sociaux relatifs à l'« usager » qui, du fait même de leur médiatisation, constituent un « diagnostic public » de l'institution et de sa qualité de service dans ce type de contexte. Nous adopterons, toutefois, dans le cadre de cet article, un bornage de corpus centrant les observations sur la journée du 3 janvier qui constitue le moment-clef de la "couverture » médiatique de cette perturbation en nous limitant à la télévision (journaux du soir).

- l'épisode en question permet de sortir de visions normatives du type : « ce que l'on voudrait que l"usager-client' soit ", ce que l'institution et son organisation en révèlent comme représentations publiques. Ces visions existent légitimement et font partie intégrante des stratégies de fonctionnement et de développement d'une entreprise publique comme la SNCF. Le dysfonctionnement de service que représente ce blocage du trafic au cours d'une des périodes les plus aiguës pour un transporteur public permet sans doute de faire apparaître, du côté du voyageur, des visions du service tel qu'il le perçoit et, corrélativement, la vision prospective d'une espèce d'«idéal de service» auquel les contraintes ponctuellement subies font songer. Les discours sur " comment l"usager-client' se voit » (et se voit traité), qu'ils émanent des voyageurs eux-mêmes ou des journalistes, se présentent donc comme des baromètres, certes très approximatifs et peu représentatifs mais indicatifs d'un climat de perception de la gestion de l'incident et, plus largement, de réception du service.

- par conséquent, les récits d'expérience ou plutôt, les bribes de ces récits que nous proposent les médias, sont autant de façons d'argumenter un point de vue sur le service ou sur ce qui, dans ces conditions particulières, en est apparu comme une défaillance. Tout récit peut donc être considéré comme une argumentation, mais sur un registre implicite (Delforce, 1999). Raconter, dans ce cas, c'est mettre en scène une part de son expérience pour la faire valoir comme un point de vue avisé - ou, du moins médiatiquement construit comme tel, puisqu'issu de la confrontation directe à la situation. La mise en mots de cette expérience ne peut alors être considérée comme une simple mise en forme mais comme un élément du système de sens, plus ou moins critique, élaboré par l'énonciateur puis réagencé, au montage et à l'écriture, par le média.

- d'un point de vue davantage organisationnel, et comme nous l'évoquions plus haut de façon plus générale, une telle situation de perturbation peut être considérée comme un révélateur des qualités ou des faiblesses structurelles de l'institution, un moment symptomatique de l'efficience du système ou des limites de celle-ci. En ce sens, tout discours produit à cette occasion apparaît comme un outil d'évaluation in situ de cette organisation, de ses potentialités d'anticipation de ce type d'incident, de systèmes de représentation des solutions trouvables (ou inenvisageables) à ces problèmes. Autant d'éléments qui peuvent 
être considérés comme partie intégrante de la qualité de service et que les médias contribuent à faire percevoir comme tels.

- enfin, nous voudrions nous interroger sur la nature du rôle du journaliste dans cette configuration informative. N'est-il pas là pour faire cœxister, dans un même espace, des discours qui ont parfois du mal à se rencontrer et qui, publicisés, n'apparaissent plus tant comme des discours individuels que comme des discours porteurs de valeurs collectives, éventuellement opposables à une parole institutionnelle (Delforce, Noyer, 1999) ? Quels antagonismes ou complémentarités de logiques nous sont ainsi proposés?

\section{La mise en scène médiatique d'un déficit de communication}

13 La circulation de l'information en interne, dans toute entreprise de transport et en particulier à la SNCF, est un élément déterminant de l'exploitation et de la coordination de l'action à tous les niveaux. C'est, par delà les moyens techniques permettant de « réaliser » le transport, un des éléments de base permettant d'accomplir ce que nous appellerons le « service premier » : celui consistant à transporter. Mais, l'information fait aussi partie des attentes de l'«usager", comme l'attestent bon nombre d'enquêtes menées par/pour l'institution: on considèrera que nous sommes, avec cet autre aspect, dans ce que nous pourrions appeler le "service second", qui concerne l'information externe, celle qui est destinée au voyageur et qui vise à construire la relation à l'« usager " à travers certains objectifs consistant à élaborer et à faire percevoir une certaine qualité de service. C'est ainsi que les annonces (dans les gares, dans les trains...) pourront fonctionner comme autant d'actes de langage tendant à manifester une attention, une volonté d'accompagnement, un certain niveau de présence marqué par l'anticipation, la prévention... A d'autres moments, il s'agira, par ce biais, de marquer un souci de coopération, de réassurance voire de sécurisation (Borzeix, 1995-b). Autant de variations discursives qui, dans la relation de service, constituent pour l'« usager » le signe, le tremplin interprétatif d'une qualité d'attention à laquelle il estime avoir droit et que la stratégie de l'entreprise publique renforce, au travers d'une politique centrée sur la relation-clientèle, étayée par une volonté d'« engagement » où personnalisation et prise en compte individualisée apparaissent non seulement comme des promesses centrales mais comme de véritables outils d'évaluation de la structure et de son management.

Les perturbations du transport ferroviaire de ce début 97 transforment les gares, habituels espaces de simple transit et de circulation de flux, en lieux de séjour de foules de voyageurs transformés en «manants» (Boullier, 96), attentifs aux signes de l'institution destinés à écourter leur attente ou, au moins, à la faire mieux comprendre et la rendre plus confortable. L'« interdit de séjour " qu'est, en principe, l'« usager » des gares est contraint au séjour forcé dans le lieu symbolique de la fluidité, ce qui le met en situation d'attente de toute information pouvant signifier une reprise du trafic ou une prise en compte de sa gêne, de son inconfort... A l'écoute de toute information, de toute annonce pouvant venir réduire son incertitude, il est aussi particulièrement disponible aux sollicitations des médias pour en mesurer l'ampleur. Captif, il l'est doublement: d'une situation de blocage et où il se trouve en état de dépendance totale; des médias ayant sous la main un public rendu disponible par cette situation et enclin à saisir l'occasion d'« envoyer un message » à l'institution? 

(transport) et l'évaluation de sa défaillance mais le service second (la délivrance d'information). Ce qui est reproché à la SNCF, ce n'est pas d'abord un déficit de technicité empêchant les TGV de rouler dans les conditions climatiques du moment, c'est un défaut de prise en considération des voyageurs, qui est lu à travers l'absence d'information à leur égard.

Vous savez ce que c'est d'être dans l'attente, sans explication? (TF1); On ne nous donne aucun renseignement (TF1); On ne sait pas si on va dormir quelque part, on ne sait rien (TF1); Le guichet des renseignements est fermé (TF1) ; Je trouve que c'est assez déplorable de laisser les gens dans ce froid, à se cailler, sans aucun renseignement (TF1) ; Ma fille, est-ce qu'elle part ce soir ou demain, on ne sait pas (F3), On se pose des questions et nous, les usagers, on est dans le noir (F3); On ne nous dit rien (F3)...

discours de l'inattention radicale de l'entreprise-SNCF au sort des voyageurs se trouve d'ailleurs, fréquemment renforcé par le niveau énonciatif complémentaire que constitue le discours du journaliste assurant le commentaire :

Ils ont le sentiment d'avoir été abandonnés, sans explication ni dialogue (TF1); Pour eux non plus, ni accueil, ni information (TF1) ; Côté renseignements, la SNCF semble toujours en panne (TF1); Pour les passagers, le pire, c'est d'attendre, de ne pas savoir ce qui va leur arriver (TF1); Certains attendent depuis le début de l'après-midi, en quête d'une information, mais en vain (F3)...

Les images de regards inquiets vers des panneaux d'affichage, qui nous sont ensuite montrés comme porteurs d'un seul type d'information qui est l'annulation des trains, viennent appuyer un premier type de version $d u$ «service second»: l'absence d'information, signifiée ici comme un manquement au contrat tacite avec l'« usager ». Ceci peut, au demeurant, être mis en relief par certains plans montrant, en parallèle avec les commentaires précédents, une affiche faisant état des engagements de service de la SNCF ou un panneau électronique sur lequel les vœux de bonne année de l'entreprise passent en boucle. Ce qu'une telle démarche informative met finalement en évidence, c'est l'inadéquation entre une qualité attendue - et matérialisée par l'engagement de service - et une qualité perçue qui, malgré la spécificité de la situation, aboutit à faire ressortir une relation défaillante, voire ce qui est considéré comme un abandon de l'« usager ». Sachant, comme le rappelle Jeannot (1998), que le marketing des services a tendance à fortement reposer sur le niveau d'engagement dans la relation, on peut imaginer les conséquences désastreuses, en terme d'image pour l'institution, d'un épisode comme celui des $2 / 3$ janvier 97 et l'effet de renforcement qu'en constitue la médiatisation fortement événementialisée. On peut, du coup, mesurer toutes les limites - et tous les risques - d'une certaine forme de communication publique jouant sur des effets d'annonce que l'institution n'est pas matériellement et structurellement prête à mettre en œuvre en toutes circonstances, y compris très particulières comme celles auxquelles nous nous attachons. Le " tout est possible, surtout le bazar " d'une voyageuse, dans un reportage de France 3, témoigne de la contreproductivité - remobilisable à chaque 
perturbation même mineure du trafic - de slogans comme celui de la campagne « SNCF, c'est possible». L'écart, toujours potentiellement présent dans une entreprise de transport, entre une promesse inconsidérée et les contre-exemples qu'on pourra en trouver sur le terrain montre bien qu'il y a quelque risque à faire transiter la communication d'une entreprise publique - notamment de transport - par de tels mots d'ordre.

\section{Sur quelques perceptions médiatisées de l'absence de qualité}

20 Un deuxième discours mobilisé par les médias consiste, en quelque sorte, en une atténuation du précédent : l'entreprise n'est plus fustigée pour son refus à communiquer (ou ce qui est interprété comme tel) mais on donne à voir et à entendre la rareté, l'indigence et, au total, l'improductivité de l'information qu'elle délivre.

A Lyon, hier soir, les guichets étaient pris d'assaut mais les employés de la SNCF bien incapables de fournir la moindre réponse (TF1); Surtout, ne pas perdre une miette d'information (F2) ; PC de crise et services d'information semblent dépassés par l'afflux des voyageurs (F3); A l'accueil, les agents sont débordés. Une seule réponse est donnée : retard indéterminé (F3).

21 Ces propos journalistiques - généralement accompagnés d'images montrant, soit des voyageurs en groupes compacts se pressant devant un guichet d'information, soit les agents d'un poste de contrôle et dont les gestes ainsi que les propos témoignent d'une maîtrise très partielle de ce qui est en train de se dérouler - laissent apparaître.

- un décalage entre la promesse faite par l'entreprise et sa réalisation effective (renforcé par la conjonction image-commentaire que nous évoquions plus haut)

- un déficit de communication externe auquel certaines images de désorganisation interne viennent donner une clef interprétative possible : celle d'un lien entre les deux.

Ce qui se trouve pointé par les médias, dans une telle situation, ce sont les phénomènes de tension et de contradiction entre une volonté stratégique - l'engagement, annoncé et revendiqué, au service du "client $»^{8}$ - et l'incapacité effective à mettre en œuvre sur le terrain ces engagements. Au lieu de fonctionner comme un élément de relativisation du jugement négatif sur la performance, la situation de crise apparaît, au contraire, comme un révélateur particulièrement signifiant des déficits de qualité. Ce qu'induisent les engagements de service comme mobilisation interne, comme modifications organisationnelles pour susciter cette mobilisation, est retraduit par le classique discours d'une forme d'inconséquence, de lourdeur structurelle générant à la fois de l'attente insatisfaite et ce qui est perçu comme de l'incohérence - alors qu'il s'agit peut-être de la prise en compte de l'évolution d'une situation :

On reçoit une information par quelqu'un qui est effectivement de la SNCF et puis, dix minutes après, il y en a un autre, qui est aussi de la SNCF et qu'on a vu avec l'autre personne d'avant qui dit autre chose (F2) ; Point de vue organisation, y a un peu à revoir, à la SNCF (F3).

Complémentaire à un tel type de représentation, un autre mode de figuration du service à l'« usager », présent notamment dans les reportages traitant de l'aspect technologique de cette perturbation, passe par le discours du temps de l'information de l'« usager » et des moyens techniques qui en permettent la réduction. 
Les TGV ont toujours reçu de l'électricité pour le chauffage et les communications. Rien n'excuse donc que les informations concernant la circulation n'aient été émises en temps réel aux passagers ulcérés (F3) comme liée à la circulation rapide de l'information et à sa disponibilité. Elle témoigne aussi d'une forme de déterminisme technologique selon lequel les moyens de transmission de l'information fonctionnant, l'information ne pouvait qu'exister, sans que son contenu et son opportunité ne puissent être considérés comme pouvant poser problème. Dans cette logique, toute absence d'information est par conséquent « inexcusable »; sauf à considérer que le paramètre technologique n'est qu'un des aspects de cette question ${ }^{9}$ et que la délivrance d'information est affaire, à la fois, de mobilisation à l'interne, de moyens (techniques et humains) pour la construire et de choix d'opportunité stratégique pour la diffuser. On voit bien, à travers ce genre de discours, à quel point une idéologie de la "transparence instantanée », liée notamment à la possibilité d'un accès immédiat à l'information, constitue une espèce de fonds perceptif commun de la relation de service, participant d'une sorte d'évidence sociale et non sujet à questionnement. Dans une telle logique, tout délai, c'est-à-dire souvent toute forme de réflexion sur les conséquences de la délivrance de l'information, risque d'être perçu comme signe d'archaïsme ou, pire, comme une forme plus ou moins grave de «manipulation » et d'irrespect de l'« usager ».

\section{La double modernisation : enjeux et décalages}

L'image publique généralement produite sur la question de la modernisation à la SNCF apparait comme essentiellement structurée autour d'« objets technologiques » de pointe, comme le TGV, et selon une logique de la performance technique, voire de la prouesse. Il est manifeste que la communication publique de l'entreprise a généré, pour partie, une perception où la modernisation de l'entreprise est davantage organisée autour du perfectionnement technologique et des performances du matériel qu'autour de la modernisation structurelle de l'organisation, fréquemment renvoyée, comme notre cas l'atteste, à une image de réactivité limitée voire d'inadaptation. Au-delà de l'entrepriseSNCF elle-même, les records de vitesse d'un train - contenant jusque dans son nom ce signe de la performance - ont permis d'accroître la renommée internationale d'une technologie nationale capable de concevoir ce type de matériel. Ils ont certes également contribué à asseoir le crédit de l'entreprise qui en assure l'exploitation mais sur un axe purement technologique. Symétriquement, il nous semble qu'ils ont contribué à forger une attente de niveau comparable sur le plan de ce que nous avons appelé le service second. La complexité d'élaboration de dispositifs organisationnels permettant de « construire ce service », notamment en situation perturbée, est à l'origine des effets de décalage entre deux modernisations dont l'une rend plus visible les aléas de l'autre.

Cette logique de complémentarité entre ces deux formes de modernisation nous semble particulièrement bien éclairée par l'épisode événementiel sur lequel nous nous sommes arrêtés. Lorsqu'un aléa climatique comme celui de janvier 97 perturbe la circulation des TGV, un double mouvement se fait jour dans le discours sur la question de la performance: parler de cette perturbation, c'est réaffirmer le niveau de performance technique du TGV, c'est prolonger le discours d'avant-crise sur la révolution technologique que représente ce type de train :

Études de communication, 23 | 2001 
Système très efficace puisqu'il a permis de battre le record du monde de vitesse (TF1); Le train le plus rapide du monde... (F2); Le fleuron électrique de la technologie française (F3) ; Une des plus belles machines du siècle (F3)... dans le discours de l'« usager » et dans celui du journaliste, comme les constantes d'un jugement qui, d'une prise de parole à une autre, varient de l'étonnement dénonciateur à la franche condamnation. L'idée sous-jacente à de telles remarques est celle d'un service de transport devant pouvoir fonctionner en toutes circonstances. Cela positionne implicitement la notion de service (premier) en termes d'absolu sans qu'elle puisse être pondérée par la prise en compte d'autres critères. La représentation de la qualité du service a tendance à s'adosser à la logique du « $\mathrm{Y}$ a qu'à... » qui, ici, consisterait en un chauffage systématique des caténaires, sans qu'en soit évoqué le coût et ses répercussions. Il nous semble, de fait, que l'on privilégie dans ce cas, chez l'« usager » du service, la face consommateur ${ }^{10}$ à celle de co-producteur ou de citoyen pour lequel la question du coût d'un tel investissement devrait être liée à son efficience. Hormis le directeur de la SNCF lui-même (sur le plateau de TF1 et dans un reportage de France 2), aucun propos (d'« usager» ou de journaliste) ne viendra mettre en perspective le coût financier important de l'investissement consistant à équiper en matériel anti-gel l'ensemble des caténaires et la faible fréquence d'utilisation d'un tel matériel.

Ce sont des centaines de millions. Faut-il dépenser des centaines de millions alors que la probabilité que cette affaire se reproduise est très faible et que nous attendrons peut-être vingt ans pour nous en resservir. Eh bien, il y a des choix à faire (L. Gallois, F2)

Pour le dirigeant de l'entreprise, le temps court de la réponse à une demande sociale, que les médias agrègent, et le temps long de la gestion prévisionnelle, liée à la direction d'une entreprise nationale, viennent se télescoper. Du coup, dans la relation au public que construit le média, deux faces du responsable de l'entreprise sont mobilisables sans qu'elles soient forcément montrées comme entrant en conflit : la face du responsable du service à l'« usager » sommé par les médias d'apporter des solutions immédiates aux problèmes qui se posent ; la face gestionnaire du même responsable, ayant à rendre des comptes devant la collectivité - et pas seulement aux «usagers» des services de l'entreprise.

Dans une situation d'urgence comme celle de janvier 97, c'est la première face qui est largement sollicitée et mise en demeure de témoigner, sur le champ, d'une capacité de mobilisation et d'organisation attestant d'une prise en charge effective de l'« usager ». 
L'autre face est, en quelque sorte, minorée voire occultée, le média s'attribuant une place d'auxiliaire, de défenseur immédiat de l'« usager» captif de la perturbation : l'urgence surdétermine la posture journalistique, calée sur la demande de secours à l'« usager » et laissant à l'écart les données gestionnaires. Les situations d'urgence étant généralement les résultantes de processus complexes, force est de constater qu'un média comme la télévision est peu enclin - sans doute parce qu'il a peu les moyens en temps, à la fois d'enquête et d'insertion dans le format réduit d'un journal télévisé - à reconstruire ces processus pour témoigner de la légitimité ou de l'illégitimité d'une décision. Ce qui est rendu visible, c'est la conséquence négative pour l'" usager ", sans que celle-ci soit reliée à un processus décisionnel dont la légitimité, en termes de gestion des ressources, peut ne pas être contestable mais que le contrat implicite d'assistance médiatique à l'« usager » occulte. Si la décision était (budgétairement) légitime, les conséquences en sont (ponctuellement) négatives - et c'est sans doute le risque qu'a à assumer n'importe quel responsable (Delforce, 1999). Mais le travail des médias, construisant l'événementialité à partir de cet écart ponctuel de fonctionnement, va générer une sorte d'«effet de focalisation " sur le dysfonctionnement et ses conséquences, induisant une prise en charge, par le média, de l'« usager » en lieu et place d'une institution montrée comme défaillante.

Dans d'autres types de situation également susceptibles de médiatisation (cf. supra), ce sera la seconde face du responsable qui pourra être sollicitée, le média se rangeant à une option gestionnaire, soucieuse de l'«argent du contribuable ». Il nous semble toutefois qu'il ne s'agit là que d'une simple variation de la version précédente par laquelle se trouve réaffirmée l'assistance à l'" usager ", pris ici, lui aussi, sous une autre face : celle de contributeur au fonctionnement de l'État. La reconstruction médiatique - et en particulier télévisuelle- de la légitimité des décisions en matière financière dans le domaine des services publics affronte en effet un double obstacle :

- la complexité du travail de reconstitution du cheminement décisionnel

- ce que l'on suppose être un intérêt limité du public pour ce type de question complexe.

En ce qui concerne plus particulièrement la SNCF, le traitement de ce problème prend la forme récurrente du déficit de l'entreprise et d'un usage de l'argent du contribuable posant question. On voit, par conséquent, en quoi la demande implicite - dans le cas que nous avons étudié - d'un équipement de chauffage systématique des caténaires vient heurter le discours d'une maîtrise raisonnée des dépenses et de dénonciation des déficits. Mais ce heurt n'est que rarement explicité par le média et manifesté comme une tension difficilement réductible entre deux demandes contradictoires d'un « usager » pouvant occuper stratégiquement et l'une et l'autre place: celle du voyageur, celle du contribuable. Que le dirigeant de l'entreprise soit seul, comme nous l'avons relevé plus haut, à pointer cette contradiction est, dès lors, susceptible d'apparaitre comme le point de vue de quelqu'un d'engagé dans la gestion de l'entreprise - et seulement cela - là où, de fait, une véritable caractéristique des logiques sociales concernant l'«usager " apparait : la variation stratégique des places - conférées ou adoptées. 


\section{L'information sur un plateau ou les multiples formes de la réparation publique}

Sous des formes diverses, la parole institutionnelle trouve une place importante dans le traitement que proposent les différentes chaînes de cet épisode de perturbation: le directeur de la SNCF, Louis Gallois, est sur le plateau de TF1 et se voit attribuer, en réponse aux questions de Claire Chazal, un temps de parole équivalent à l'ensemble des reportages sur cette question (environ 5'). Sur France 2, ses propos sont inclus dans le reportage de Romuald Bonnant. En ce qui concerne France 3 et étant donné les caractéristiques de la chaîne, le choix est fait d'axer la couverture de l'épisode sur la région sud-est et de donner la parole au responsable Rhône-Alpes (Bernard Véron).

Nous souhaiterions, dans cette dernière partie, montrer que ce que nous avons appelé plus haut la « relation de service médiatisée » fait partie intégrante, par l'intermédiaire de ces prises de parole publiques, de la gestion d'une crise comme celle de janvier 97 et s'articule autour d'une série d'actes qui, par le biais du média, prennent valeur d'actes particulièrement symboliques contribuant à l'image publique de l'entreprise. On peut d'ailleurs considérer que le média, un peu comme le «front office » pour l'agent, est ce lieu, ce front auquel le responsable doit monter en pareille circonstance pour assurer publiquement l'ensemble de ces actes témoignant d'une volonté de réparation (Joseph, 1988) auprès des « usagers » et du public, dans sa globalité.

Comme dans tout rapport de places (Watzlawick, Beavin, Jackson, 1972, Flahaut, 1978), la place adoptée par le dirigeant est d'abord une place contrainte par celle que prend l'interlocuteur et, en l'occurrence, les questions que posent les journalistes apparaissent généralement comme une parole déléguée des «usagers » bloqués ou de ceux qui les attendent. En fonction de la relation du média au public évoquée plus haut, le média se positionne comme relais, comme médiateur d'une attente, d'une demande des « usagers ». La place du responsable, induite par celle de ce journaliste-porte-parole, est donc essentiellement défensive, réactive par rapport à un cadre qui lui est imposé et dont elle ne peut que difficilement sortir ; ce qu'elle parvient toutefois à faire lors de tentatives de recadrage de l'importance du problème comme dans cette remarque de Louis Gallois où, en filigrane, réapparait le conflit entre logique médiatique de focalisation sur l'écart et logique institutionnelle :

Je rappelle que nous avons fait circuler 1000 trains hier; il y en a eu 30 de bloqués.

Alors, c'est 30 de trop mais il y en a 970 qui ont circulé.

Une telle démarche de relativisation, si elle est publiquement adressée et donc à usage externe pour moduler l'image médiatique de déficit de qualité, nous apparaît être également à usage interne et donc implicitement adressée au public de l'entreprise. S'opère ici ce que l'on pourrait appeler un mouvement de "défocalisation", visant à élargir le prisme étroit d'attention des médias dans ce genre d'épisode et à affirmer publiquement, sur cette base, la reconnaissance d'une activité et d'une compétence au sein de l'institution lorsqu'elles sont questionnées par ces mêmes médias. La prestation télévisée du dirigeant - ou du responsable régional - est donc, d'une certaine façon, un acte de « réparation interne » par lequel celui qui dirige évacue ce qui, dans le traitement médiatique, pourrait apparaitre comme une dénonciation implicite du travail des agents. Le média apparaît ainsi comme le vecteur de diffusion d'un message de solidarité à 
l'interne, dont l'affirmation publique est une double forme d'engagement: auprès des membres de l'entreprise, auprès du public de l'entreprise.

Nous ne sommes pas parfaits, c'est vrai. Mais ce que je peux dire, c'est que les agents de la SNCF, les cheminots ont fait tout ce qu'ils ont pu, ils se sont véritablement défoncés. Je voulais le dire parce qu'on ne l'a pas suffisamment dit et je voulais leur rendre hommage. Ils ont fait un travail formidable (Louis Gallois, TF1); Tous les cheminots qui sont là se dévouent pleinement et montrent leur bonne volonté et leur professionnalisme pour aider les voyageurs à poursuivre leur voyage malgré des conditions très difficiles (Bernard Véron, F3) ; Nos équipes techniques, qui travaillent jour et nuit, vont tenter de déglacer les fils de contact... (BV, F3) ; Nos équipes continuent de travailler d'arrache-pied (BV, F3).

Il est clair, par ailleurs, que cet acte de réparation, pour s'exercer publiquement avec la crédibilité qu'un dirigeant d'entreprise peut en attendre, a tendance à passer par une série de "démarches explicatives» qui sont autant d'éléments de négociation d'une image de l'entreprise (et de son dirigeant) face au public.

La première de ces démarches consiste en une opération d'auto-évaluation dont la version globale serait le «nous ne sommes pas parfaits » cité plus haut et qui concerne la perturbation de service dans sa plus large extension. Sur le plan plus spécifique de ce que nous avons appelé le service second, la déclaration, dans la même séquence-plateau, de "nous ne sommes pas des communicants extraordinaires" participe de la même démarche auto-évaluatrice de reconnaissance d'un déficit de qualité. La nature concessive d'une telle démarche montre bien qu'elle est inscrite dans le cadre d'une évaluation préalable : celle proposée par le média à partir de l'ensemble des discours verbaux et iconiques développés. La concession dans l'appréciation des responsabilités liées à l'épisode se fait, on le remarquera, avec le secours de la litote qui, sans doute, permet une forme de protection publique des faces de l'institution - aussi bien celle de l'entreprise comme communauté que celle de sa direction.

Le jugement négatif que l'on concède à l'interlocuteur et à l'ensemble du public est néanmoins relié à une opération conjointe d'autojustification qui participe de la démarche plus large de relativisation de la responsabilité. Celle-ci s'opère, notamment, par une série d'explications mettant en avant l'impossibilité d'un message à communiquer ("Nous ne savions pas ce que nous pouvions leur dire ", LG, TF1) là où, apparemment, l'attente des « usagers " (cf. leurs propos directs dans les reportages) était dans la manifestation d'une relation, d'un contact, de signes d'accompagnement du voyageur en difficulté - même sans contenu informatif manifeste. Le non-savoir sur les possibilités de trafic, lié aux aléas climatiques mais formulé comme une incapacité à dire ( "Je suis incapable de vous dire si les trains rouleront demain », LG, TF1) laisse ouvertes toutes les réinterprétations possibles en termes de failles organisationnelles, de mauvaise circulation de l'information, de stratégies d'évitement de l'« usager »...

Dans de telles circonstances - dont le caractère «exceptionnel » fonctionne comme un des arguments de l'autojustification - le média va avoir tendance à adopter une posture ${ }^{11}$ d'auxiliaire de service, se substituant à ce qui a été construit comme la défaillance de l'entreprise. C'est ainsi que s'opère, dans l'interaction sur le plateau, ce que nous appellerons une " pression à l'information-service ", de nature immédiatement utilitaire, où le responsable - ainsi mis en position basse - est sommé de faire preuve de réactivité et d'adaptation aux circonstances. Cette pression à l'information-service aura généralement comme résultat des actes de promesse, de conseil, de réassurance ayant pour vocation à la fois la manifestation d'une attention spécifique à l'« usager » et la 
régulation ultérieure du trafic. Mais, l'incapacité où se trouvera Louis Gallois de donner, en réponse à la demande pressante de Claire Chazal, le numéro vert d'assistance aux « usagers » ou à leurs familles fonctionnera, dès lors, comme un élément de confirmation du diagnostic critique du média à l'égard de l'institution, atténuant du même coup le travail de réparation antérieurement mis en œuvre par le directeur de la SNCF.

Les médias et, en particulier, la télévision, recueillent les réactions immédiates liées à un problème dans une situation et mettent en visibilité de façon non moins immédiate ces réactions (Mouchon, 1997). Ces réactions sont elles-même liées à ce que l'institution rend visible/audible par le biais des annonces, du télépancartage, de l'accueil... La perception du service (second: d'information) est donc étroitement liée aux démarches, aux choix institutionnels de mise en visibilité de telle ou telle information. On voit bien, par conséquent, que l'acte institutionnel de mise en visibilité par le biais de l'accueil et des moyens d'annonce orale et/ou écrite est tout à fait déterminant dans la relation à l'«usager» mais aussi dans la perception du niveau de qualité de ce service par cet " usager ». Que les médias, à partir de leurs critères propres de sélection de ce qui mérite médiatisation (cf. supra) choisissent de mettre en visibilité tel déficit, tel manquement de l'institution-SNCF dans son contrat avec l'« usager » - client, - tel défaut de présence et d'accompagnement dans le cas que nous avons étudié - et c'est l'institution dans sa globalité et l'intégralité de sa politique qui, par delà les démarches d'évolution structurelle et de modernisation au jour le jour, sont renvoyés à l'image d'un archaïsme persistant. Il nous semble, par conséquent, qu'une entreprise comme la SNCF et, plus généralement, le secteur des services (aux) publics ne sauraient faire l'économie d'une réflexion précise sur les logiques de médiatisation dans lesquelles ils sont pris, des choix de cadres - notamment discursifs - mobilisés au sein de ces logiques et des types de faces et figures des acteurs qui s'y trouvent plus systématiquement convoqués.

\section{BIBLIOGRAPHIE}

Borzeix, Anni, (1995-a), « Un rouage ultime : l'annonce sonore », in Gare du Nord, mode d'emploi, Éditions Recherches, Plan Urbain, RATP-SNCF, Paris.

Borzeix, Anni, (1995-b), «Qualité et bienveillance », in Métiers du public, I. Joseph, G. Jeannot (dir.), CNRS Éditions, Paris.

Borzeix, Anni, Fisher, Sophie, De Fornel, Michel, Lacoste, Michèle, (1995), « Les lettres de réclamation » in L'Administration de l'Équipement et ses usagers, C. Quin (dir.), La Documentation Française, Paris.

Boullier, Dominique, (1996), « Les automates de Montparnasse : les transactions, les agents... et les usagers? ", Les annales de la recherche urbaine, $\mathrm{n}^{\circ} 71$.

Chauvière, Michel, Godbout, Jacques, (dir.), (1992), Les usagers entre marché et citoyenneté, L'Harmattan, Paris.

Cosnier, Jacques, Picard, Dominique, (1992), La relation de service en station, ARCI, RATP Prospective, Paris. 
Daviet, Jean-Pierre, (1996), « Le service public et l'usager, entre le droit administratif et la philosophie politique (1873-1945) », in Consommateur, usager, citoyen : quel modèle de socialisation?, C. Horellou-Lafarge (dir.), L'Harmattan, Paris.

Delcambre, Pierre, (1998), « Penser des pratiques culturelles en se saisissant du concept de médiation », Études de Communication (Médiations culturelles : dispositifs et pratiques), $\mathrm{n}^{\circ} 21$, Université Lille 3.

Delforce, Bernard, (1999), Pour une approche interdisciplinaire des phénomènes de médiatisation : la dimension discursive, Mémoire en vue de l'habilitation à diriger des recherches, Université Lille 3.

Delforce, Bernard, Noyer, Jacques, (1999), Pour une approche interdisciplinaire des phénomènes de médiatisation : constructivisme et discursivité sociale, in Études de Communication (La médiatisation des problèmes publics), $\mathrm{n}^{\circ} 22$, Université Lille 3.

Flahaut, François, (1978), La parole intermédiaire, Éditions du Seuil, Paris.

Grémion, Catherine, Fraisse, Robert, (dir.), (1996), Le service public en recherche : quelle modernisation?, Commissariat général au plan, La documentation française, Paris.

Grosjean, Michèle, (1993), «L'informateur au PCC : transporteur ou stratège ? ", in Régulation du trafic et information des voyageurs au PCC de la ligne A du RER, Isaac Joseph (dir.), RATP Prospective, Paris.

Heath, Christian, (1993), « Diagnostic et consultation médicale : la préservation de l'asymétrie dans la relation entre patient et médecin ", in Soins et communication, J. Cosnier, M. Lacoste, M. Grosjean (dir.), ARCI, Presses Universitaires de Lyon.

Horellou-Lafarge, Chantal, (dir.), (1996), Consommateur, usager, citoyen : quel modèle de socialisation?, L'Harmattan, Paris.

Jeannot, Gilles, (1998), Les usagers du service public, P.U.F., Paris.

Joseph, Isaac, (1988), « La relation de service : les interactions entre agents et voyageurs », Les annales de la recherche urbaine, $\mathrm{n}^{\circ}$ 39, Ministère de L'Équipement et du Logement, Éditions Dunod.

Lacoste, Michèle, (1995), « Les locaux d'information », in Gare du Nord, mode d'emploi, Éditions Recherches, Plan Urbain, RATP-SNCF, Paris.

Mouchon, Jean, (1997), « Visibilité médiatique et lisibilité sociale », in La communication de l'information, Jean-Pierre Esquenazi (dir.), L'Harmattan, Paris.

Quéré, Louis, (1995), « L'espace public comme forme et comme événement », in Prendre place, Isaac Joseph (dir.), Éditions Recherches, Plan Urbain, Paris.

Quin, Claude, (dir.), (1995), L'Administration de l'Équipement et ses usagers, La Documentation Française, Paris.

Warin, Philippe, (1992), « Décrire la conduite des usagers », in Les usagers entre marché et citoyenneté, M. Chauvière, J. Godbout (dir.), L'Harmattan, Paris.

Watzlawick, Paul, Beavin, J., Helmick, Jackson, Don, D., (1972), Une logique de la communication, Éditions du Seuil, Paris. 


\section{NOTES}

1. La systématicité des guillemets pour cette notion dans cet article est une façon de pointer, sous le choix de la forme dénominative que nous adopterons, les logiques diverses - et parfois antagonistes - qui creusent une telle notion (Chauvière-Godbout, 1992).

2. La variété des types de « services» - et des types de «fonctions" - rendent toute globalisation difficile en matière de services publics: la forme plurielle adoptée voudrait rendre compte de cette diversité (Grémion-Fraisse, 1996).

3. Parmi lesquelles les médias, et en particulier la télévision, peuvent jouer un rôle notable mais non exclusif. On renverra, pour un parcours du concept et des formes de la médiation, à la postface de Pierre Delcambre au numéro 21 d'Études de Communication consacré aux médiations culturelles.

4. Si les entretiens avec la « clientèle » et les questionnaires de satisfaction réalisés dans le cadre d'enquêtes-marketing peuvent jouer un rôle dans ces évolutions, on aurait tort de sous-évaluer sous prétexte de son insuffisance en terme de représentativité - la prise en compte attentive des paroles d'« usagers " recueillies par d'autres biais, notamment en cas d'incidents mobilisant l'attention des médias.

5. L'éventail des logiques de médiatisation et des situations-types exposées ici ne saurait correspondre qu'à une partie des cas de figure sur la question que nous travaillons.

6. Les institutions de services publics, dans ce qu'il est convenu d'appeler la communication de crise, se chargent généralement de réinstiller de la programmation de leur action - et de sa couverture médiatique - pour faire apparaître leur compétence de réactivité et d'adaptabilité, construisant par ce biais leur image, au-delà de leur stricte communication publique. EDF, de ce point de vue, est un bon exemple.

7. Une pleine page de publicité paraîtra dans l'ensemble de la presse quotidienne nationale, le 10 janvier 97, sous le titre «Message reçu ». S'y trouvaient annoncés les engagements de la SNCF consécutifs à cet épisode de perturbation.

8. L'adoption progressive à la SNCF d'une logique d'entreprise induite par la concurrence intermodale en matière de transport a eu comme conséquence désignative systématique la substitution de « client » à « usager ».

9. On se reportera, sur cette question, à l'article de Bruno Raoul dans ce numéro.

10. Au sens de la stricte consommation d'un produit qui est ici le transport, dans une logique de droit systématique à l'usage.

11. Pour la notion de " posture ", nous renvoyons à Delforce-Noyer (1999), « Pour une approche interdisciplinaire des phénomènes de médiatisation : constructivisme et discursivité sociale», Études de Communication $n^{\circ} 22$.

\section{RÉSUMÉS}

La question traitée par l'article que nous proposons est celle de l'usager du service public et de la relation de service tels qu'ils sont reconfigurés par les médias et, en particulier, la télévision au cours d'une situation de crise touchant le transport ferroviaire, dans la vallée du Rhône, durant l'hiver 96-97. L'auteur s'interroge sur le problème de l'information en situation perturbée, sur les 
représentations qui en sont médiatiquement produites et sur les conceptions de la modernisation du service public auxquelles elles renvoient. Il envisage, parallèlement, la place qu'un média tel que la télévision s'assigne dans une telle configuration et les usages, notamment discursifs, qu'en font les différents acteurs.

This paper addresses the issue of users of public service and of the notion of service as they were reconfigured by the media, most particularly, by television during a crisis situation affecting rail transportation in the Rhône Valley during the winter of 1996-97. The paper considers the issue of information in a disrupted situation, the representations that are generated by the media, and the conceptions of the modernization of public service these reflect. It also addresses the space a medium such as television allows itself in such a configuration as well as the uses, especially discursive ones, the different actors make of it.

\section{INDEX}

Mots-clés : usager, service public, analyse du discours, média, médiatisation, communication institutionnelle

Keywords : user, public service, speech analysis, media, media coverage, corporate communication

\section{AUTEUR}

\section{JACQUES NOYER}

Jacques Noyer est Maître de Conférences en Sciences de l'Information et de la Communication à l'Université Charles-de-Gaulle - Lille 3. Il est membre de l'équipe « Espace public et médias » (GERICO - Lille 3). Ses recherches portent sur le rôle et la place des médias et de leurs discours dans la construction des problèmes publics. Il y privilégie l'approche en termes de processus de médiatisation envisagé comme logiques discursives autant que comme logiques d'acteurs. 